\title{
GTSE1 expression represses apoptotic signaling and confers cisplatin resistance in gastric cancer cells
}

Vinod Vijay Subhash', Shi Hui Tan², Woei Loon Tan², Mei Shi Yeo², Chen Xie², Foong Ying Wong², Zee Ying Kiat ${ }^{2}$, Robert Lim² and Wei Peng Yong ${ }^{1,2^{*}}$

\begin{abstract}
Background: Platinum based therapy is commonly used in the treatment of advanced gastric cancer. However, resistance to chemotherapy is a major challenge that causes marked variation in individual response rate and survival rate. In this study, we aimed to identify the expression of GTSE1 and its correlation with cisplatin resistance in gastric cancer cells.

Methods: Methylation profiling was carried out in tissue samples from gastric cancer patients before undergoing neoadjuvent therapy using docetaxel, cisplatin and 5FU (DCX) and in gastric cancer cell lines. The correlation between GTSE1 expression and methylation in gastric cancer cells was determined by RT-PCR and MSP respectively. GTSE1 expression was knocked-down using shRNA's and its effects on cisplatin cytotoxicity and cell survival were detected by MTS, proliferation and clonogenic survival assays. Additionally, the effect of GTSE1 knock down in drug induced apoptosis was determined by western blotting and apoptosis assays.

Results: GTSE1 exhibited a differential methylation index in gastric cancer patients and in cell lines that correlated with DCX treatment response and cisplatin sensitivity, respectively. In-vitro, GTSE1 expression showed a direct correlation with hypomethylation. Interestingly, Cisplatin treatment induced a dose dependent up regulation as well as nuclear translocation of GTSE1 expression in gastric cancer cells. Knock down of GTSE1 enhanced cisplatin cytotoxity and led to a significant reduction in cell proliferation and clonogenic survival. Also, loss of GTSE1 expression caused a significant increase in P53 mediated apoptosis in cisplatin treated cells.
\end{abstract}

Conclusion: Our study identifies GTSE1 as a biomarker for cisplatin resistance in gastric cancer cells. This study also suggests the repressive role of GTSE1 in cisplatin induced apoptosis and signifies its potential utility as a therapeutic target for better clinical management of gastric cancer patients.

Keywords: GTSE1, Drug resistance, Cisplatin, Apoptosis

\section{Background}

Despite of significant advances in therapeutic strategies and a decline in incidence over the last few decades, gastric cancer still remains as the second most leading cause of cancer-related mortality worldwide $[1,2]$. Currently, surgery represents gold standard for the

\footnotetext{
* Correspondence: Wei_Peng_YONG@nuhs.edu.sg

${ }^{1}$ Cancer Science Institute of Singapore, National University of Singapore, Singapore, Singapore

${ }^{2}$ Department of Haematology-Oncology, National University Hospital of Singapore, Singapore, Singapore
}

treatment of gastric cancer without distant metastasis. However, chemotherapy appears to be a useful option in the treatment of advanced gastric cancer, with a modest but real survival benefit [3]. Platinum based chemotherapy containing cisplatin and oxaliplatin were shown to have promising results with similar response rate and progression-free survival (Cunningham et al.; 2008). Although platinum based therapy is highly active in gastric cancer, a marked individual variation in response rate (RR) and survival rate is seen among patients undergoing treatment. In order to better control the local relapse and increase in survival time of advanced patients, the role of neoadjuvant chemotherapy 
(NAC) is currently being investigated with different protocols. Multiple phase II and phase III trials utilizing docetaxel, cisplatin and 5-FU (DCX) have shown this combination to be highly effective, particularly in advanced gastric carcinoma $[4,5]$. Albeit these advances, the appearance of drug resistance limits the effectiveness of cancer chemotherapy and poses a major impediment in clinical treatment [6]. Earlier studies have revealed the major mechanisms underlying resistance that include reduced uptake and/or increased efflux and enhanced DNA repair $[7,8]$. As tumors are highly adaptable, drug resistance can also be induced by the activation of survival signaling pathways and the inactivation of downstream death signaling pathways [9]. Additionally, epigenetic changes, changes in the molecular phenotype and the influence of the local tumor microenvironment, could also play contributory roles in chemoresistance [10]. Hence, elucidating the mechanism underlying the sensitivity and resistance to chemotherapy is critical to develop a more personalized approach towards treatment of gastric cancer.

Human GTSE1 (G2 and S phase expressed-1) is expressed specifically during $\mathrm{G} 2$ and $\mathrm{S}$ phases of the cell cycle, and is localized mainly in the cytoplasm, associated with microtubules [11]. GTSE1 is cell cycle regulated and becomes phosphorylated in mitosis and markedly reduced in $G_{1}$ phase of cell cycle [12]. Over expression of GTSE1 results in a delay of the $G_{2}$ to $M$ phase transition [13]. The protein is reported to shuttle between the cytoplasm and nucleus, however it gets stabilized in the nucleus following DNA damage. Once in the nucleus, GTSE1 acts as a negative regulator of $\mathrm{p} 53$ expression where it binds and relocalizes p53 to the cytoplasm to undergo degradation [14]. Consequentially, the DNA damage induced transactivation of p53 is inhibited, thus affecting p53 induced apoptosis $[14,15]$. In the absence of DNA damage, GTSE1 has been reported to localize to the interphase microtubule networks where it exists in association with clathrincontaining complexes [16, 17]. Tian et al. (2011) have shown that GTSE1 is up-regulated in lung cancer tissues compared to the adjacent normal tissues, especially in adenocarcinoma and squamous cell carcinoma. Of interest, a more than two-fold increase in GTSE1 expression was shown in myeloma cells after cisplatin treatment, suggesting a mechanism of clinically acquired drug resistance [18].

This study explored the expression, cellular localization and functional significance of GTSE1 in gastric cancer. GTSE1 methylation was found to be associated with better treatment response to DCX- chemotherapy in gastric cancer patients. A correlation between GTSE1 expression and cisplatin cytotoxicity is suggested here, as cisplatin treatment induced a dose dependent up regulation of GTSE1 in gastric cancer cells. This increase in expression was seen associated with a change in cellular localization as well. Intriguingly, loss of GTSE1 expression contributed to enhanced cisplatin sensitivity and p53 induced apoptotic signaling in gastric cancer cells. Taken together, by identifying the regulatory role of GTSE1 in cisplatin sensitivity and drug induced apoptosis, this study signifies the potential implications of GTSE1 as a biomarker for cisplatin resistance in gastric cancer. Moreover, our study presents an additional candidate for personalised molecular targeted therapy that could overcome cisplatin resistance and thereby attempts to improve the therapeutic index of this compound in clinical applications.

\section{Methods}

\section{Analysis of microarray datasets}

Two independent microarray datasets (Gastric cancer: a, GSE13911; b, GSE27242) of pair wise tumor tissues and adjacent normal tissues were retrieved from www.oncomine.org and the mRNA expression level of GTSE1 was investigated. A total of 169 and 69 samples were analysed in GSE27242 and GSE27242 respectively.

\section{Patient recruitment and study design}

21 consecutive patients with locally advanced [AJCC TNM (T3/4 or N+ M0)] histologically-proven gastric or esophagogastric adenocarcinoma with no evidence of distant metastases, or locally advanced inoperable disease, as evaluated by computed tomography (CT), chest radiography, ultrasonography, or laparoscopy were included in the study. Pre-treatment characteristics of patients are mentioned in Table 1. DCX combination was administered in a 21-day cycle for three cycles before surgery. The first cohort of 10 patients received intravenous docetaxel $35 \mathrm{mg} / \mathrm{m}^{2}$, intravenous cisplatin $35 \mathrm{mg} / \mathrm{m}^{2}$ on day 1 and day 8 , with oral capecitabine $750 \mathrm{mg} / \mathrm{m}^{2}$ twice daily from day 1 to day 14 . A subsequent cohort of another 11 patients had dose modifications to docetaxel $30 \mathrm{mg} / \mathrm{m} 2$, cisplatin $30 \mathrm{mg} / \mathrm{m}^{2}$ and capecitabine $700 \mathrm{mg} /$ $\mathrm{m}^{2}$ due to high rates of diarrhea in the first cohort of 10 patients Preoperative radiological response was evaluable in 17 patients after two cycles of chemotherapy (Additional file 1: Figure S1). GTSE1 methylation was determined in 19 patients prior to neo-adjuvent chemotherapy. A total of 14 patients underwent curative surgery and the surgical details are mentioned in Additional file 1: Table S1. Seven patients did not undergo surgery with three having withdrawn consent and two declined surgery after completing neoadjuvant chemotherapy and two had disease progression. Eleven patients underwent post treatment pathological assessment. The median follow-up was 25 (23-27) months after surgery and patients were classified as responders or non-responders by the radiological response (Responders: complete response or nearcomplete response; Non-responders: partial response or others). The median follow-up was 25 (23-27) months after surgery and patients were classified as responders or 
non-responders by the radiological response (Responders: complete response or near-complete response; Nonresponders: partial response or others). The protocol was reviewed and approved by the institution's review board and informed consent was obtained from each patient. Ethical approval was obtained from NHG (National Health Group) Domain Specific Review Board, Singapore.

\section{Genomic methylation profiling}

Genomic DNA from primary gastric tissues (11) and cell lines (39) were bisulphite-modified using EZ DNA methylation kit (Zymo Research). The modified samples were profiled in infinium $27 \mathrm{~K}$ methylation array (Illumina). DNA methylation levels for each CpG site were computed by Genome Studio software as the ratio of methylated intensity to the sum of methylated and unmethylated signal intensities.

\section{Cell culture and drug treatment}

AZ521 and OCUM-1 were obtained from Japanese Collection of Research Bioresources, Japan. SNU610 and SNU719 were purchased from Korean Cell Line Bank, Korea. All the cell lines were cultured at $37{ }^{\circ} \mathrm{C}$ in a humidified atmosphere containing $5 \% \mathrm{CO}_{2}$ and maintained in RPMI 1640 medium (Gibco), containing $10 \%$ heat inactivated fetal bovine serum (Gibco) and $1 \%$ penicillin/ streptomycin (Gibco). For dose response studies, the cell lines were treated with cisplatin at IC50 concentration.

\section{Cell Synchronization}

Cells were synchronized at the $\mathrm{G}_{1} / \mathrm{S}$ border by treating with $2.5 \mathrm{mM}$ thymidine for $16 \mathrm{~h}$ followed by extensive wash and release into normal growth medium for $10 \mathrm{~h}$ to obtain cells in $\mathrm{G}_{2} / \mathrm{M}$. The cell cycle stage was monitored by staining with propidium iodide $(10 \mu \mathrm{g} / \mathrm{ml})$, followed by flow cytometric analysis performed on a $\mathrm{BD}^{\mathrm{TM}}$ LSR II (BD biosciences) equipped with FlowJo software (version vX 0.7).

\section{Quantitative PCR (qPCR) analysis}

Total RNA was extracted from cultured cells with RNeasy Mini kit (Qiagen), with the use of QIAshredder spin column for homogenization and an on-column DNase digestion. $2 \mu \mathrm{g}$ of the total RNA was reversely transcribed using M-MLV reverse transcriptase enzyme (Promega). The cDNA obtained was analysed quantitatively using Power SYBR Green PCR Master Mix (Applied Biosystems) on an ABI7300 Real-time PCR system. Primers used are listed in Table 2. Cycling conditions were $95{ }^{\circ} \mathrm{C}$ for $15 \mathrm{~min}, 40$ cycles of $15 \mathrm{~s}$ at $94{ }^{\circ} \mathrm{C}, 30 \mathrm{~s}$ at $55{ }^{\circ} \mathrm{C}$ and $30 \mathrm{~s}$ at $72{ }^{\circ} \mathrm{C}$. Ct values were generated using default analysis settings. Relative quantification (RQ) was calculated using $2^{-\Delta \Delta C T}$ method.
Table 1 Pre-treatment characteristics of patients

\begin{tabular}{ll}
\hline Pre-treatment characteristics of patients, $n=21$ & \\
\hline Characteristic & \\
Median (yr) & $61(32-77)$ \\
Sex-no. (\%) & \\
Male & $16(76.2 \%)$ \\
Female & $5(23.8 \%)$ \\
ECOG performance status- no. (\%) & \\
0 & $18(85.7 \%)$ \\
1 & $3(14.3 \%)$ \\
Clinical staging (EUS/CT staging) & \\
T1 & $0(0 \%)$ \\
T2 & $2(9.5 \%)$ \\
T3 & $17(81.0 \%)$ \\
T4 & $2(9.5 \%)$ \\
No & $7(33.3 \%)$ \\
N+ & $14(66.7 \%)$ \\
Histology grade & \\
Moderately differentiated & $3(14.3 \%)$ \\
Poorly differentiated & $18(85.7 \%)$ \\
\hline
\end{tabular}

Bisulphite conversion and methylation-specific polymerase chain reaction (MSP)

DNA was extracted from the gastric carcinoma cell lines with Puregene ${ }^{\text {TM }}$ DNA Isolation Kit (Gentra Systems). 500 ng of the cell line DNA, positive control DNA (CpGenome Universal Methylated DNA, Chemicon) and negative control Human Sperm DNA (HsD) were used for bisulphate conversion using EZ DNA Methylation-Gold ${ }^{\mathrm{TM}}$ Kit (Zymo Research Corporation) as per manufacturer's protocol. Primers used for MSP reactions are listed in Table 3. PCR was performed by preheating at $94{ }^{\circ} \mathrm{C}$ for $5 \mathrm{~min}$, then 40 cycles of denaturation at $94{ }^{\circ} \mathrm{C}$ for $30 \mathrm{~s}$, annealing at $56{ }^{\circ} \mathrm{C} / 56{ }^{\circ} \mathrm{C}$ for $60 \mathrm{~s}$ for methylated/unmethylated GTSE1, and extension at $72{ }^{\circ} \mathrm{C}$ for $60 \mathrm{~s}$, followed by a final 7 min extension at $72{ }^{\circ} \mathrm{C}$. The PCR products were separated on a $2 \%$ agarose gel.

\section{Small hairpin (sh) RNA transfection}

Human GTSE1 'SureSilencing shRNA' plasmids were purchased from Qiagen. AZ521 cells $(1 \times 10 \mathrm{e} 5$ cells/ well) were plated in 6-well plates and grown in $2 \mathrm{ml}$ RPMI-1640 medium with $10 \%$ FBS. Transfections of GTSE1 shRNA and control vectors were performed using X-treme gene transfection reagent (Roche) according to the manufacturer's instructions. Independent colonies were isolated by ring cloning, and expanded in $1000 \mu \mathrm{g} / \mathrm{ml}$ neomycin. Cell lysates were collected and GTSE1 expression was detected by western blotting. 
Table 2 RT PCR primer sequences used to detect GTSE1 mRNA expression

\begin{tabular}{ll}
\hline RT-PCR primers & Sequence \\
\hline GTSE1 forward & 5-GCC CCG GGT GCT GTC AAT GT-3 \\
GTSE1 reverse & 5-GCC CAC TGC TGG GGA TGT GC-3 \\
GAPDH forward & 5'-TGA AGG TCG GAG TCA ACG GAT TTG GT-3 \\
GAPDH reverse & 5'-CAT GTG GGC CAT GAG GTC CAC CAC-3' \\
\hline
\end{tabular}

\section{MTS- cell viability assay}

To assess the chemosensitivity of tumor cells to cisplatin, cell viability was measured by MTS (Colorimetric CellTiter 96 Aqueous One Solution Cell Proliferation Assay) (Promega). Cell suspension was cultured in 96-well flatbottomed microtiter plates at seeding density of $2 \times 10 \mathrm{e} 3$ cells/well and incubated overnight. Drug treatments were carried out at a dilution range of 0.01-1000 $\mu \mathrm{M}$ cisplatin. Microtiter wells containing tumour cells with no drug treatments were used as controls, and wells containing complete medium were used as blank controls. Cells were incubated for $72 \mathrm{~h}$ before the addition of MTS solution $(1 \mathrm{mg} / \mathrm{mL}$ per well) and absorbance was read at $550 \mathrm{~nm}$ using a spectrophotometric microplate reader (Bio-Rad). The percentage cell viability at different drug concentrations was calculated as the inhibition rate of (mean absorbance of treated wells/mean absorbance of control wells) $\times 100 \%$. IC50 was calculated by GraphPad Prism v4.0 (GraphPad Software, Inc).

\section{Cell proliferation (BrdU) assay}

Cells were seeded on to 96 -well plates at a density of $2 \times 10^{3}$ cells/well. Cell proliferation was measured using the BrdU proliferation assay (Roche) at 12, 24 and $48 \mathrm{~h}$ according to the manufacturer's protocol. The absorbance values are directly correlated to the amount of DNA synthesis.

\section{Colony formation assay}

The ability for colony formation at low cell density was determined by plating $1 \times 10^{3}$ cells/well onto a 6 -well plate and then culturing for 7 days. Cells were subsequently stained with $0.5 \%$ crystal violet in $30 \%$ ethanol and $3 \%$ formaldehyde for $10 \mathrm{~min}$ at room temperature.
Stained colonies were counted under the microscope by selecting a total of five random fields per sample.

\section{Apoptosis assay}

Apoptosis was detected by Annexin V-FITC (fluorescein isothiocyanate) kit (BD Pharmingen) according to manufacturer's instructions. Briefly, the cells $\left(1 \times 10^{5}\right.$ cells $\left./ \mathrm{ml}\right)$ were grown to $80 \%$ confluency in $25 \mathrm{~cm}^{2}$ flasks in F12K supplemented with $10 \%$ fetal calf serum. After $24 \mathrm{~h}$ of incubation with or without drug, cells were harvested, washed thrice with cold PBS and resuspended in $1 \times$ binding buffer. An aliquot of $100 \mu \mathrm{l}$ of the cell suspension was transferred into a microfuge tube and mixed with equal volumes $(5 \mu \mathrm{l})$ of Annexin V- FITC and Propidium Iodide (PI). The cells were gently vortexed and incubated for $15 \mathrm{~min}$ at $37{ }^{\circ} \mathrm{C}$ in the dark, before the addition of $400 \mu \mathrm{l}$ of $1 \mathrm{X}$ binding buffer in each tube. The cell samples were then analysed using a flow cytometer $\mathrm{BD}^{\mathrm{m}}$ LSR II (BD biosciences) equipped with FlowJo software (version vX 0.7).

\section{Protein extraction and western blot analysis}

Cells were washed with ice cold PBS and whole cell lysates were prepared using CelLytic buffer (Sigma-Aldrich). The cytoplasmic and nuclear protein fractions were extracted using NE-PER Nuclear and Cytoplasmic Extraction kit (Thermo Scientific) as per manufacturer's instructions. $20 \mu \mathrm{g}$ of protein was electrophoretically separated on $12 \%$ SDS-PAGE. Western blots were performed with the primary antibodies: anti-GTSE1 (Santa Cruz biotechnology), anti-p53, anti-Bax, anti-p21 Waf1/Cip1 (12D1), antiGAPDH (Cell Signaling) and the corresponding secondary antibodies: anti-rabbit/mouse IgG, HRP-Linked (Cell Signaling). The signals were visualized by ECL reagent (Amersham "' ECL Plus Western Blotting Detection System; GE Healthcare), followed by exposure to chemiluminescence film (Amersham Hyperfilm "' ECL; GE Healthcare). Immunoblot analyses were repeated twice for each protein tested.

\section{Statistical analysis}

Two-tailed student's $t$-test was used for differential comparison between two groups. $P$ value $<0.05$ was considered statistically significant. Differential methylation

Table 3 MSP primer sequences used to detect GTSE1 DNA methylation

\begin{tabular}{ll}
\hline Methylation/ Unmethylation primers & Sequence \\
\hline Methylated GTSE1 forward & 5'-GTA GTG CGT ATG CGT ATT GGA C-3' \\
Methylated GTSE1 reverse & 5'-GCG AAT TAC CGA TTA ATC GAT-3' \\
Unmethylated GTSE1 forward & 5'-AGT AGT GTG TAT GTG TAT TGG ATG-3' \\
Unmethylated GTSE1 reverse & 5'-AAA ACA CAA ATT ACC AAT TAA TCA AT-3' \\
\hline
\end{tabular}


analyses between 2 groups were performed using the statistical package in R (www.r-project.org).

\section{Results}

GTSE1 is highly expressed in primary gastric tumours and correlates with hypomethylation in gastric cancer cells

In order to verify the expression level of GTSE1 mRNA, we analyzed the public microarray database, Gene Expression Omnibus (Gastric cancer: a, GSE13911., b, GSE27242). GTSE1 was up-regulated in both datasets with a fold change of 4.07 and 3.34 and $t$-test $\mathrm{p}$-value $7.37 \mathrm{E}-10$ and $1.72 \mathrm{E}-6$ respectively.
Figure 1a shows the $\log 2$ transformed fold change of GTSE1 mRNA level in tumor tissue versus nontumor in GSE13911. These findings were further supported by investigating GTSE1 mRNA expression in gastric cancer cell lines. As shown in Fig. 1b, GTSE1 expression in 4 gastric cancer cell lines- AZ521, SNU60, OCUM-1 and SNU719 was analysed by qPCR. All the cell lines showed detectable expression of GTSE1 mRNA, among which AZ521 showed a relatively high GTSE1 expression, whereas SNU 719 showed a relatively low expression. Furthermore, the association between expression and methylation status

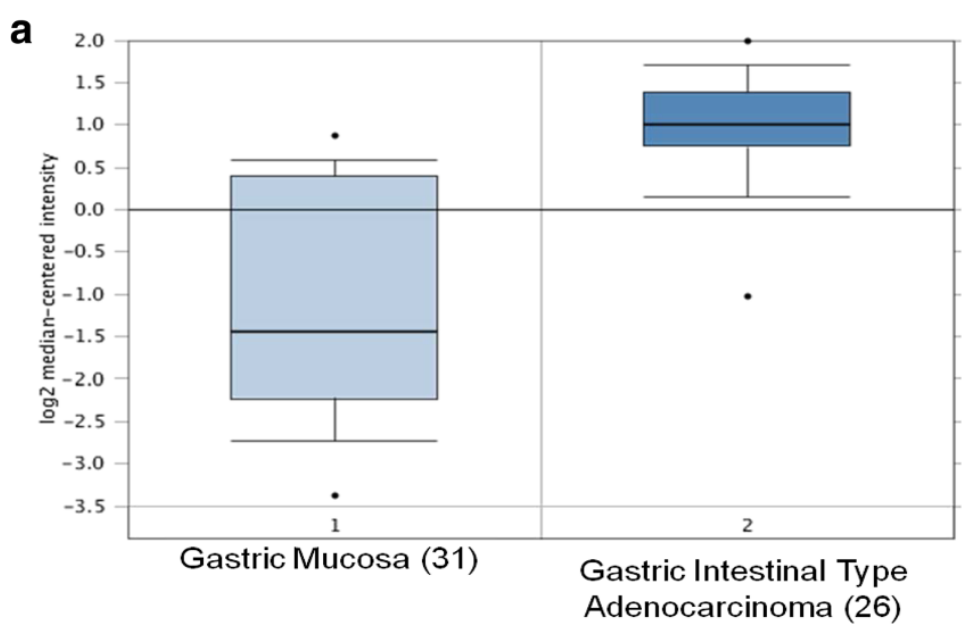

b
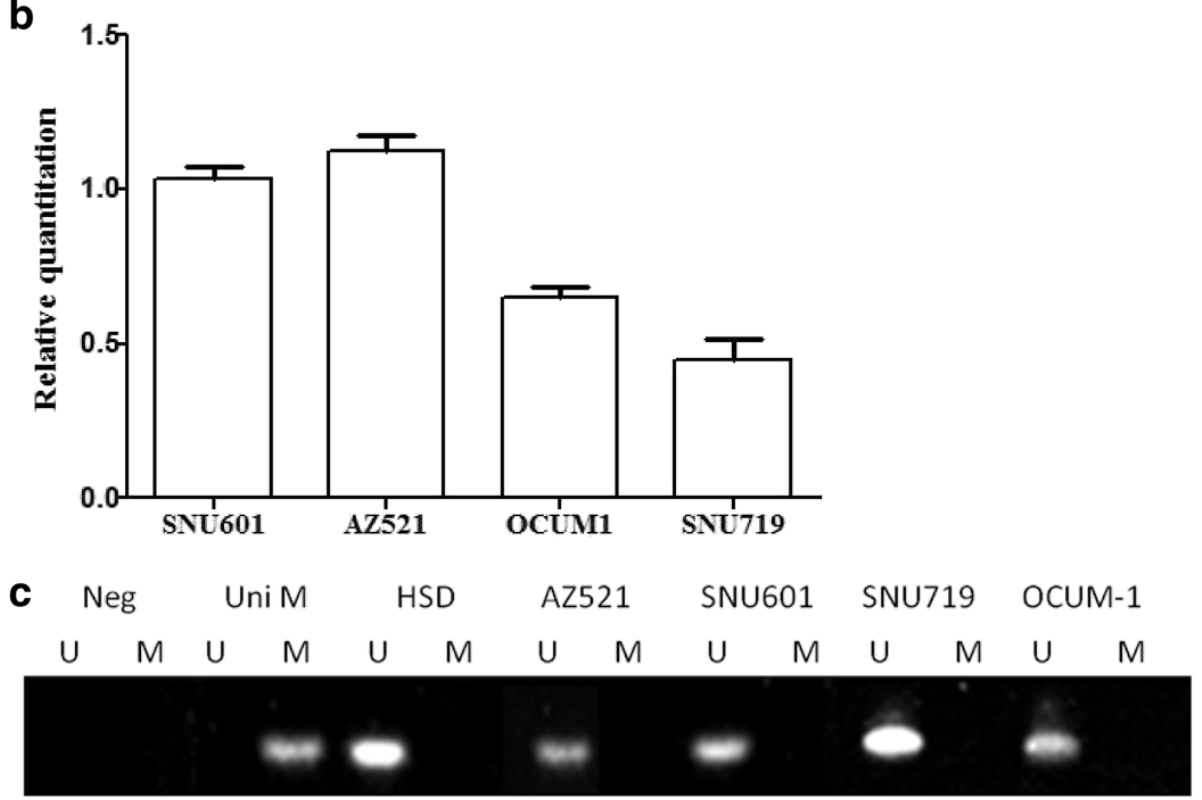

Fig. 1 a GTSE1 Log2 transformed expression level. Fold change of each sample between tumor tissues and non-tumorous tissues in microarray from GEO in gastric cancer (GSE13911) is shown. $\mathbf{b}$ The mRNA expression of GTSE1 in 4 gastric cancer cells detected by qPCR. The gene expression of GTSE1 was normalised relative to GAPDH as internal control. The $\mathrm{p}$ value shown for the average data was computed using a paired two-tailed Student $t$ test $(n=3)$. Error bars denote standard deviations. c DNA promoter methylation status of MAGE-A1 in 4 gastric cell lines by MSP. UniM: Universal methylated DNA is the control sample for methylation; HSD: human sperm DNA is the control sample for unmethylation. M: methylation; U: unmethylation 
was determined by MSP (Fig. 1c). A $100 \%$ (4/4) concordance was observed between GTSE1 hypomethylation and expression as all the cell lines were unmethylated for GTSE1.

\section{Differential methylation of GTSE1 correlates with treatment response to DCX and Cispaltin sensitivity}

Methylation profiling of tissue samples obtained from 11 GC patients before receiving DCX treatment depicted hypomethylation of GTSE1 (range: 0.03-0.08). GTSE1 was shown to be differentially methylated in GC patients who are responders to DCX combinational chemotherapy. Statistical analyses revealed that GC patients who were classified as responders $(n=3)$ by pathological response have significantly higher GTSE1 methylation $(\mathrm{T}=4.01, p=0.04)$ as compared to non-responders $(\mathrm{n}=8)$ (Fig. 2a). Similarly, methylation profiling of $39 \mathrm{GC}$ cell lines also suggested hypomethylation of GTSE1 (range: 0.01-0.08). Statistical analyses performed on the top 10 cisplatin-sensitive and top 10 cisplatin-resistant cell lines revealed significantly higher GTSE1 methylation in the sensitive cell lines as compared to the resistant ones (Fig. $2 \mathrm{~b}: \mathrm{T}=3.11, p=0.006$ ). Conversely, no association was found between GTSE1 methylation and drug sensitivity of gastric cancer cells to docetaxel and 5FU (Additional file 1: Figure S2).

\section{GTSE1 knockdown enhanced cisplatin sensitivity in \\ gastric cancer cells}

The association between GTSE1 expression and chemoresistance to cisplatin was defined. GTSE1 was stably knocked-down in AZ521 cells by transfection with targeted shRNA's. Transfected variants of AZ521 cells (AZ521-kd) showed a significant reduction in GTSE1 protein expression compared to the cells transfected with the scrambled control shRNA (AZ521-cont) and the parental cell lines (AZ521-p) (Fig. 3a). To detect the role of GTSE1 in chemo resistance, the dose response of AZ521 cells to cisplatin treatment was analysed. Interestingly, loss of GTSE-1 expression contributed to enhanced sensitivity to cisplatin treatment as shown by a $\sim 5$ fold decrease in IC50 values of AZ521-kd cells $(0.8 \mu \mathrm{M})$ compared to the parental counterpart $(3.7 \mu \mathrm{m})(p=0.004)$ (Fig. 3b). The study suggests GTSE-1 to play a major role in cisplatin resistance in gastric cancer cells. The drug resistance showed by GTSE1 expressing cells appeared highly specific to cisplatin treatment, as similar dose response studies using docetaxel and 5FU in GTSE1 knocked-down cells yielded no significant variations in IC50 (Additional file 1: Figure S3).

\section{GTSE1 knock down enhanced cisplatin cytotoxicity by} causing a reduction in proliferation and colony formation of gastric cancer cells

The cytotoxic effects of cisplatin on the proliferation of AZ521 cells were measured by BrdU cell proliferation

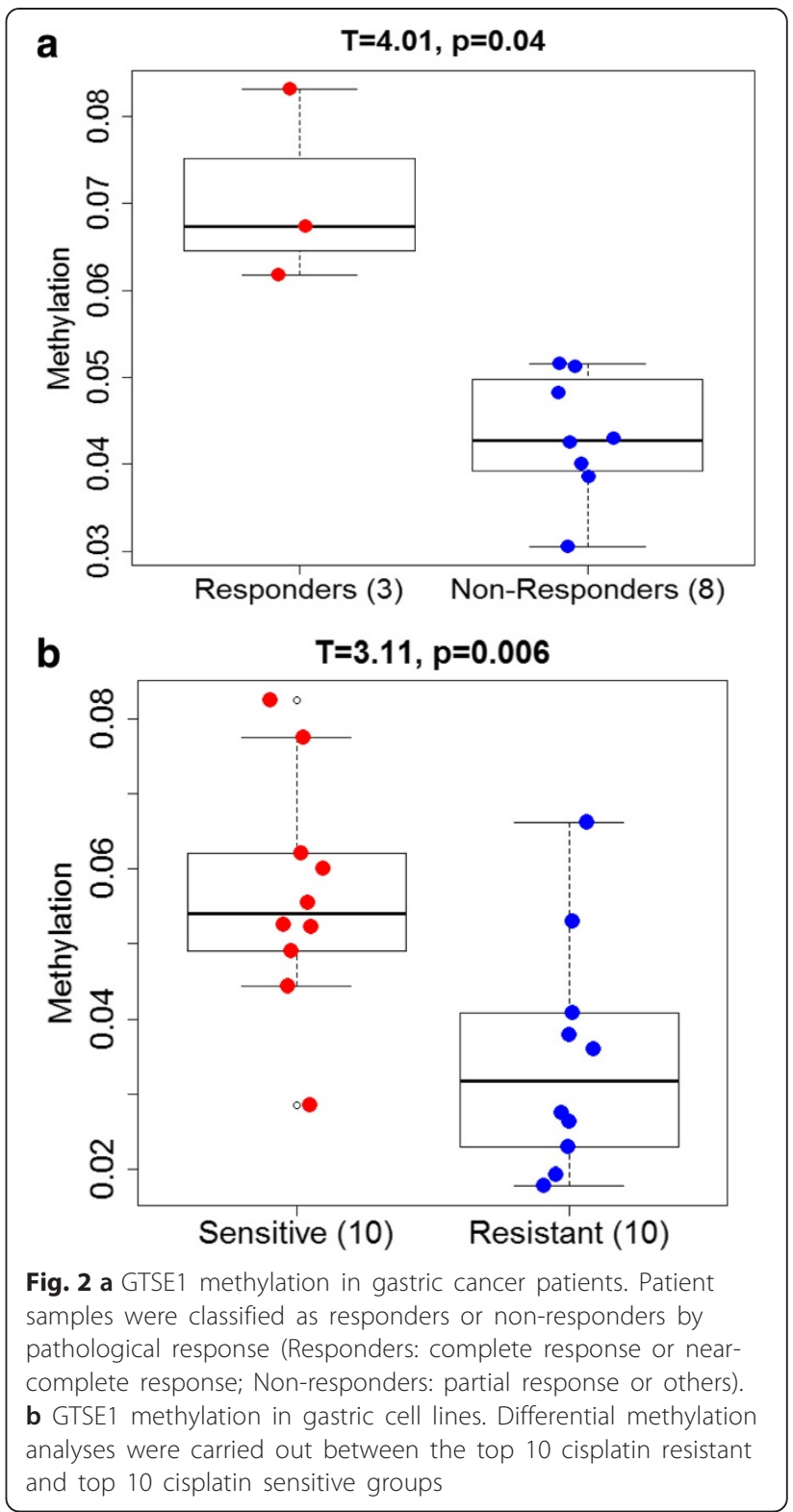

assay (Fig. 4a). Cells pre-treated with cisplatin for 12, 24 and $48 \mathrm{~h}$ showed a time dependent reduction in overall proliferation as compared to the untreated cells. Interestingly, GTSE1 knock down resulted in a greater reduction in cell proliferation as AZ521-kd cells exhibited a sharp decline in proliferation within $24 \mathrm{~h}$ of cisplatin treatment $(p=0.01)$. The results were more significant at $48 \mathrm{~h}$ of drug treatment, as observed by a fivefold decrease in proliferative cells $(p=0.003)$. To further confirm the enhanced cytototoxic effects of cisplatin in GTSE1 knock down cells, a clonogenic survival assay was performed. Although cisplatin treatment induced an inhibitory effect in the colony formation ability of both AZ521-con and AZ521-kd cells, the decrease of colony formation in GTSE1 knocked-down cells appeared to be 


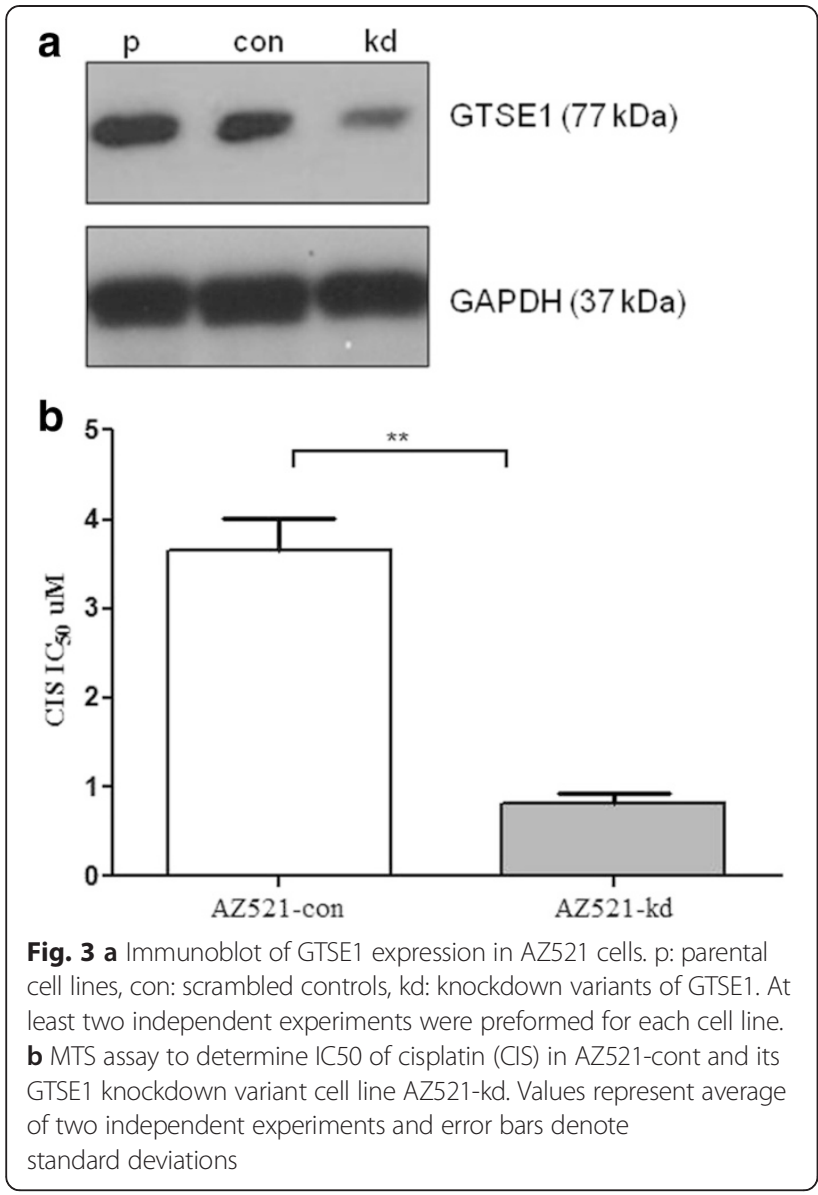

more drastic and highly significant $(p=0.002)$ as compared to the control cells (Figure $4 \mathrm{~b}$ ). Taken together, our findings suggest a role of GTSE1 in enhancing the growth and survivability of gastric cancer cells where it acts antagonistic to the cellular sensitivity towards cisplatin.

\section{Cisplatin cytotoxity upregulates GTSE1 expression and induces its nuclear translocation}

Previous studies have shown an increase in GTSE1 expression in response to DNA damage insults (Monte $e t$ al., [11], [14]). Consistently, the present study observed a dose dependent up regulation of GTSE1 expression in cisplatin treated AZ521 cells (Fig. 5a). The expression levels peaked at close to IC50 concentration of cisplatin. Moreover, a change in GTSE1 subcellular localization was also observed as cisplatin treatment induced the translocation of GTSE1 from the cytoplasm to the nucleus in a time dependent manner. As shown in Fig. 5b GTSE1 expression appeared predominantly cytoplasmic in untreated AZ521 cells. Upon $8 \mathrm{~h}$ of cisplatin treatment, a significant reduction in GTSE1 expression was observed in the cytoplasmic fraction whereas a
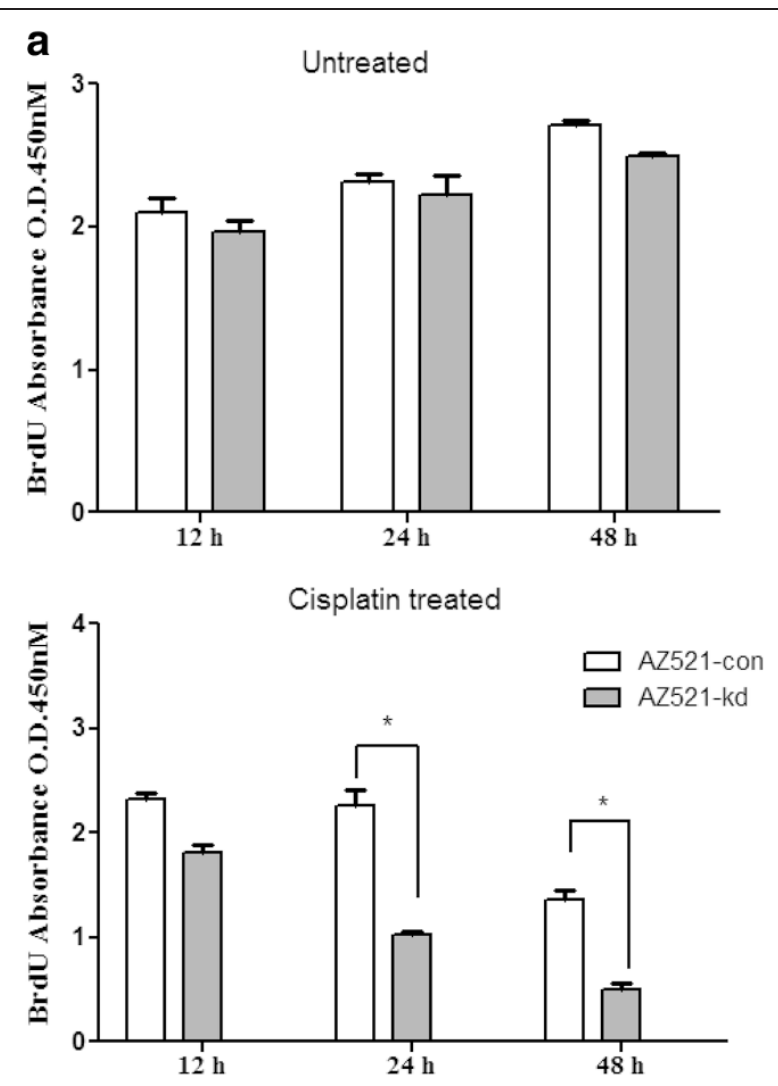

b

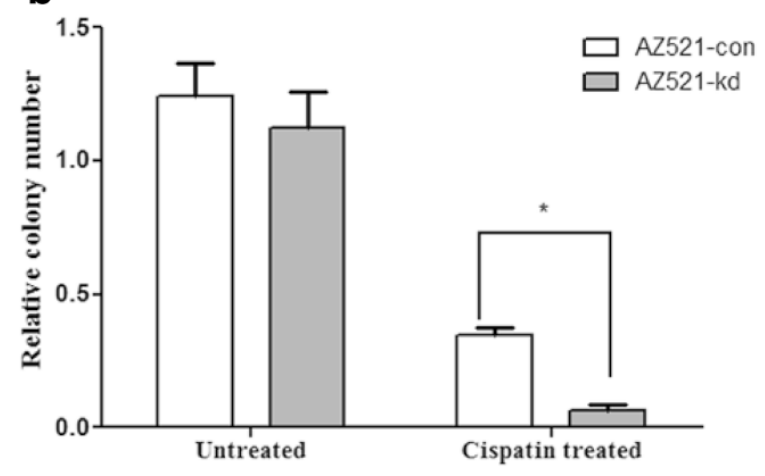

Fig. 4 a Cell proliferation assay. Proliferation rate of AZ521-con and AZ521-kd cells as evaluated with the BrdU assay at 12, 24 and $48 \mathrm{~h}$ post cisplatin treatment. Untreated cells served as experimental control. Results are representative of two independent experiments and error bars indicate standard deviations. b Clonogenic survival assay. The colony formation ability of AZ521-con and AZ521-kd cells were measured after cisplatin treatment. Untreated cells served as experimental control. Results are representative of two independent experiments and error bars indicate standard deviations

corresponding increase in expression was seen in the nuclear fractions. These findings are in accordance with and add further credence to the studies by Monte et al. [14] that showed nuclear accumulation of GTSE1 during DNA damage. 


\section{GTSE1 expression down regulates cellular apoptosis by repressing $\mathrm{p} 53$ signaling}

Studies have shown that p53 expression is up regulated upon chemotherapeutic stress and GTSE1 was previously characterised as a negative regulator of p53 (Scolz et al., [15]; Zhan et al., 2014). This prompted us to further investigate the mechanism of cisplatin sensitivity in GTSE1 knocked-down gastric cancer cells. Treatment of AZ521 cells with cisplatin caused an up regulation of p53 expression and its downstream effector bax. Interestingly, knock down of GTSE1 expression lead to a further increase in p53 and bax expression levels. This increase in expression in GTSE1 knock down cells appeared specific to cisplatin cytotoxity as untreated cells did not show any corresponding variation in p53 and bax levels (Fig. 6a). Treatment of AZ521 cells with cisplatin also resulted in caspase 3 cleavage suggesting induction of apoptosis (Additional file 1: Figure S4).On the other hand, a down regulation in p21 levels was observed in GTSE1 knocked-down cells. This down regulation of p21 converges with the previous shown functionality of GTSE1, where it prevents proteosome

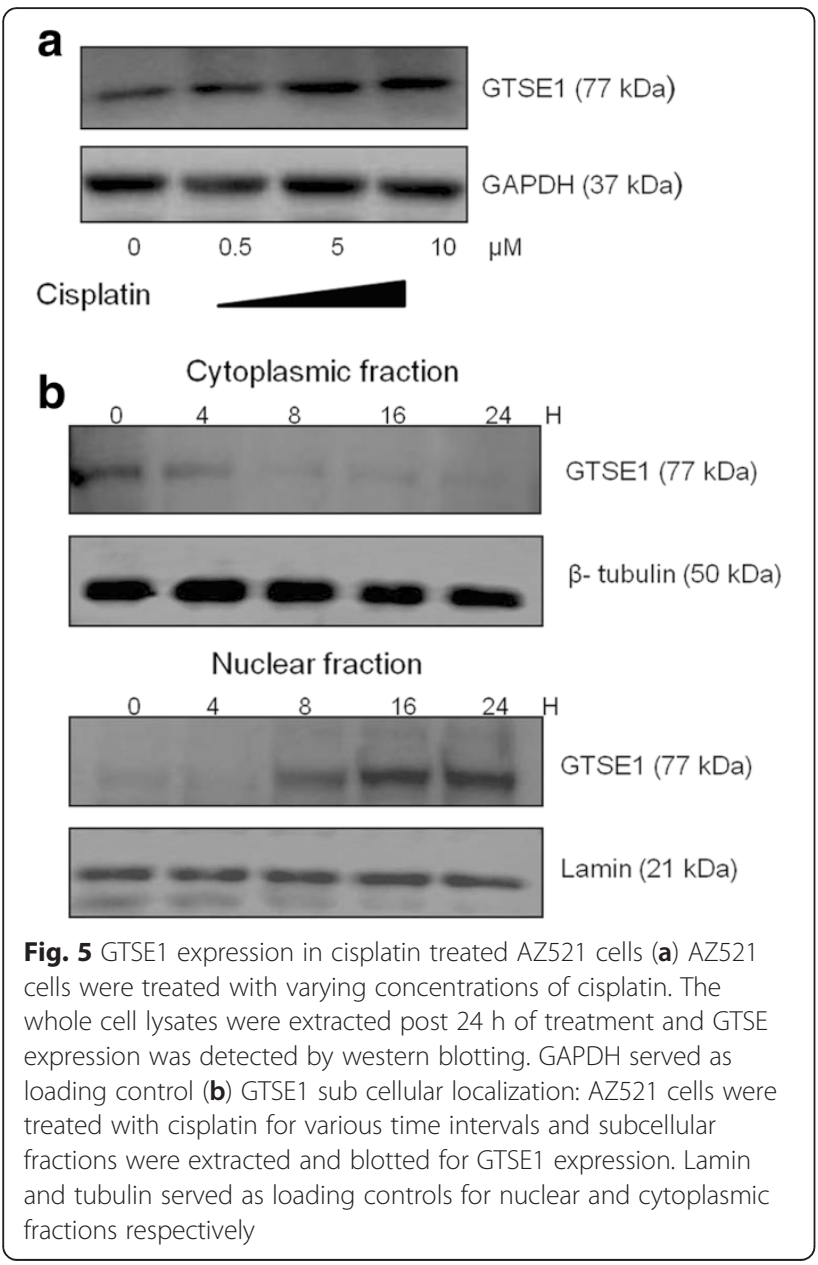

dependent degradation of p21. Notably, the biological consequence of p53 upregulation in GTSE1 knockeddown cells is also reflected by a significant up regulation in cisplatin induced apoptosis. As shown in Fig. 6b, GTSE1 knock down enhanced the sensitivity of AZ521 cells to cisplatin treatment resulting in a $>50 \%$ increase in apoptosis compared to the parental cells $(p=0.010)$.

\section{Discussion}

The use of modern genomic, proteomic and functional analytical techniques has resulted in a substantial increase in our ability to identify novel genes and signaling networks that are involved in determining the responsiveness of tumors to a particular drug treatment. The mechanisms of resistance to 'classical' cytotoxic chemotherapeutics and to therapies that are designed to be selective for specific molecular targets share many features, such as alterations in the drug target, activation of pro-survival pathways and ineffective induction of cell death [9]. Pre-clinical and clinical studies have already shown that combining information from more than one molecular biomarker increases our ability to predict tumour drug response [19]. Hence, understanding how tumors evolve on a molecular level to overcome the cytotoxic effects of chemotherapy is a critical step in developing therapeutic approaches that will prevent or overcome chemoresistance [20].

Alterations in gene expression profiles and epigenetic changes are known to be early events in the multi-steps of carcinogenesis [21]. On an epigenomewide basis, multiple DNA methylation changes in the cancer methylome have been reported to occur during the acquisition of drug resistance [22]. Here, we explored the contributory role of cell cycle dependent protein GTSE1 in gastric cancer chemoresistance. GTSE1 is highly expressed in cancers and was shown to be clinically associated with drug resistance [18]. Analysis of public gene array databases showed high GTSE1 expression in gastric cancer [23-25]. Since aberrant DNA methylation as a marker of platinumresistance was shown in lung and ovarian cancer cell lines [26, 27]; we examined the methylation status of GTSE1 in gastric cancer patients who underwent neoadjuvant DCX-combinational chemo therapy. Although GTSE1 appeared hypomethylated in both responders and non responders of DCX treatment, a clear distinction could be seen between the two groups wherein responders showed a higher methylation index. However, the current study was unable to show any significant survival benefit of GTSE1 methylation in gastric cancer patients. This could be due to the small sample size and thus hinders evaluation of GTSE1 methylation as a predictive biomarker for DCX treatment response. Consistently, GTSE1 was shown to be hypomethylated in gastric cancer cell 

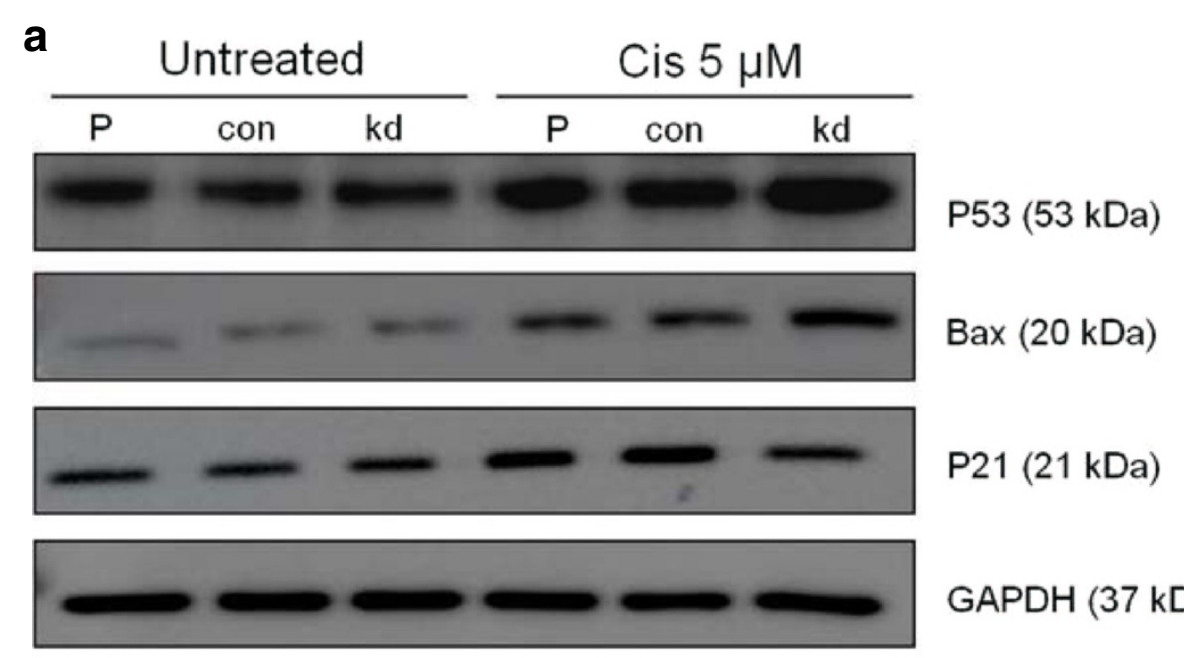

GAPDH (37 kDa)

b
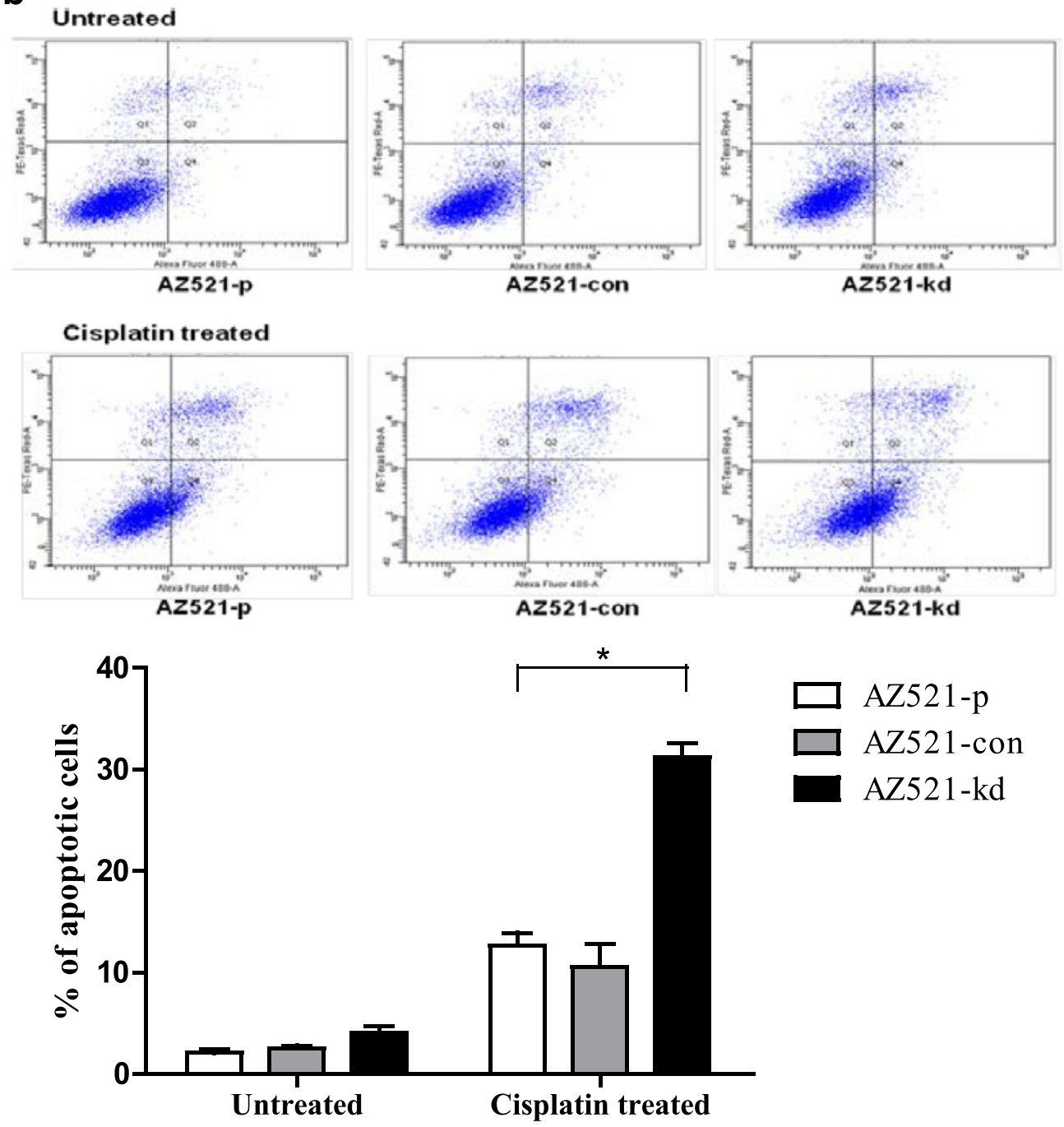

AZ521-kd

Fig. 6 (See legend on next page.) 
(See figure on previous page.)

Fig. 6 Role of GTSE1 in cisplatin induced apoptosis. a AZ521-p, AZ521-con and AZ521-kd cells were treated with cisplatin (5 $\mu \mathrm{m})$ for $24 \mathrm{~h}$ and expression of pro-apototic p53 and its downstream effectors bax and casp-3 were detected by western blotting. Untreated cells served as experimental control. GAPDH served as internal control (b) The induction of apoptosis in AZ521-p, AZ521-con and AZ521-kd as detected by flow cytometry using Annexin V-FITC apoptosis assay. The percentage of apoptotic cells (lower right quadrant) was calculated. Data are presented as the mean values of the florescent intensities from two independent experiments and error bars standard deviations (SD)

lines. All hypomethytlated cell lines showed significant expression levels of GTSE1. Intriguingly, cell lines that are sensitive to cisplatin treatment showed a significantly different methylation index for GTSE1. Since, the predominant model of regulation of gene expression underscores an inverse correlation between DNA methylation and expression [28], the higher methylation index of GTSE1 in DCX responders and in cisplatin sensitive cell lines could be suggestive of its lower expression levels. However, this study did not investigate this correlation in the DCX patient cohort. Hence, clinical implications of GTSE1 methylation status needs further investigation regarding the possible rationales raised in this study

Gene expression profiling in cancer have provided substantial information on the oncogenic potential of genes controlling essential pathways and other cellular events. Cell proliferation and growth inhibition are tightly regulated processes which are key in the maintenance of normal cell growth homeostasis and viability [29]. However deregulation of these processes occur during tumor development and is often contributory to drug resistance. The present study demonstrated a major role of GTSE1 in conferring cisplatin resistance as knock down of GTSE1 expression in gastric cancer cells enhanced cisplatin sensitivity. Moreover, cisplatin treatment induced a dose dependent upregulation as well as nuclear translocation of GTSE1 in gastric cancer cells. The increase in expression and change in membrane localization of GTSE1 falls in line with a previous study that showed up regulation and nuclear import of this protein in response to various DNA- damaging agents [14]. The nuclear translocation of GTSE1 is also suggestive of the cellular response to cisplatin induced DNA damage stress, where its expression counteracts with drug sensitivity. Enhanced proliferation and colony formation ability of cells are well associated with malignant transformation in many cancers [30]. Although anti-cancer therapy targets apoptosis of hyper-proliferative cells, its efficacy may vary according to cancers and its molecular sub-types. In our study, loss of GTSE1 expression enhanced the antiproliferative and growth-inhibitory effects of cisplatin in gastric cancer cells. Of note, the decrease in cell proliferation and colony formation observed in GTSE1 knockeddown cells is also suggestive of its tumorogenic potential that could be utilized in targeted therapies in gastric cancer. Consistent with these observations, expression of GTSE1 was found inhibitory to cisplatin cytotoxicity as its knock down lead to a two-fold increase in apoptosis of gastric cancer cells. This increase in apoptosis could well be explained by the observed increase of p53 expression in GTSE1 knocked-down cells. P53 is a potent inhibitor of cell growth and its function is tightly controlled to allow normal growth development [31]. In response to DNA damage insults, p53 induces cell cycle arrest and activates the intrinsic apoptotic signaling pathway [32]. P53 up regulation also resulted in activation of its downstream effectors- bax. This could attribute to chromatin condensation, DNA fragmentation and finally apoptosis [33]. Hence, up regulation of p53 and its downstream effectors in cisplatin treated cells can be inferred as a cellular response to DNA damage. Therefore, by identifying a reduced expression profile of p53 in normal cells against GTSE1 knocked-down cells, our study demonstrates a role of GTSE1 in attenuating p53 mediated apoptotic response in cisplatin treated gastric cancer cells. However, we did not see any significant difference in caspase 3 activation in GTSE1 knock down cells upon cisplatin treatment, as compared to its parental counterparts (Additional file 1: Figure S4). Our findings suggest that GTSE1 mediates a caspase 3 independent cascade of apoptotic repression in cisplatin treated gastric cancer cells. This is consistent with previous studies by and Cui et al. and $\mathrm{Hu}$ et al. that have shown a p53 dependent but caspase 3 independent mechanism of apoptosis [34, 35]. In addition, GTSE1 expression was also seen to be associated with high expression of p21 as knockdown of GTSE1 caused a reduction in p21 turn over. This falls in agreement with studies by Bublik et al. that showed GTSE1 as a regulator of $\mathrm{p} 21$ stability by protecting it from proteosome-dependent degradationEarlier, the association of GTSE1 with p21 was shown to modulate cellular response to paclitaxel induced apoptosis in cervical cancer cells [36]. Moreover, high levels of p21 in cancers have been linked to poor prognosis [37]. Thus, by oppositely regulating p53 and p21, GTSE1 protein may display a combined role in promoting cell survival by shifting the equilibrium of p53 response from apoptosis to survival [23]. Hence, targeting GTSE1 in cancer therapy could enhance a p53 mediated pro-apoptotic response with a reflective decrease in p21 induced cell cycle arrest.

\section{Conclusion}

Individualisation of therapy according to the molecular phenotype of tumour and patient could dramatically increase the effectiveness of chemotherapy. The study presented here emphasizes the predictive value of GTSE1 
as a biomarker for cisplatin resistance in gastric cancer. Although previous studies in lung cancer patients did not show any correlation of GTSE1 with clinical data, the drug sensitivity profile and down-regulation of p53 induced apoptotic signaling in GTSE1 knocked-down gastric cancer cells is intriguing and warrants an in depth analysis of its clinical significance. Future studies should utilize animal models to further explore the therapeutic utility of GTSE1 in gastric cancer. Nevertheless, our study identifies a previously uncharacterized role of GTSE1 in conferring cisplatin resistance and presents an additional avenue for future therapeutic intervention and patient stratification in gastric cancer.

\section{Additional file}

\begin{abstract}
Additional file 1: Table S1. Surgical results. Figure S1. Radiological response after 2 cycles of chemotherapy $(n=17)$. Figure S2. GTSE1 methylation in gastric cell lines (a) Differential methylation analyses were carried out between the top docetaxel resistant and docetaxel sensitive groups. (b) Differential methylation analyses were carried out between the top 5FU resistant and 5FU sensitive groups. Figure S3. MTS assay to determine IC50 of (a) docetaxel (DOC) and (b) 5FU in AZ521-cont and its GTSE1 knockdown variant cell line AZ521-kd. Values represent average of two independent experiments and error bars denote standard deviations. Figure S4. Caspase 3 cleavage in cisplatin treated AZ521 cells. a) AZ521-p, AZ521-con and AZ521-kd cells were treated with cisplatin $(5 \mu \mathrm{m})$ for $24 \mathrm{~h}$ and caspase 3 expression was detected by western blotting. Untreated cells served as experimental control. GAPDH served as loading control. (DOC $710 \mathrm{~kb}$ )
\end{abstract}

\section{Competing interests}

The authors declare that they have no competing interests.

\section{Authors' contributions}

WS performed the experiments. SHT performed analysis of clinical data. WLT, MSY, XC, FYW participated in experimental planning and execution. WS compiled the data and wrote the manuscript. ZWK, RL and YWP conceived of the study, participated in its design and coordination, and helped to draft the manuscript. All authors read and approved the final manuscript.

\begin{abstract}
Acknowledgements
This work was supported by NMRC Translational Clinical Research Grant, NMRC Individual Research Grant and Duke-NUS Graduate Medical School Faculty Funds sponsored by the Ministry of Health, Ministry of Education, and Ministry of Trade, Singapore and A*STAR. The authors would like to thank Cancer Science Institute of Singapore (CSI) for the research facilities and support.
\end{abstract}

Received: 30 April 2015 Accepted: 14 July 2015

Published online: 25 July 2015

\section{References}

1. Bilici A. Treatment options in patients with metastatic gastric cancer: current status and future perspectives. World J Gastroenterol. 2014;20:3905-15.

2. Siegel R, Naishadham D, Jemal A. Cancer statistics, 2013. CA Cancer J Clin. 2013:63:11-30.

3. Cunningham D, Allum WH, Stenning SP, Thompson JN, Van de Velde CJ, Nicolson $\mathrm{M}$, et al. Perioperative chemotherapy versus surgery alone for resectable gastroesophageal cancer. N Engl J Med. 2006;355:11-20.

4. Ajani JA, Fodor MB, Tjulandin SA, Moiseyenko VM, Chao Y, Cabral Filho S, et al. Phase II multi-institutional randomized trial of docetaxel plus cisplatin with or without fluorouracil in patients with untreated, advanced gastric, or gastroesophageal adenocarcinoma. In J Clin Oncol. 2005;23(United States):5660-7.
5. Roth AD, Fazio N, Stupp R, Falk S, Bernhard J, Saletti P, et al. Docetaxel, cisplatin, and fluorouracil; docetaxel and cisplatin; and epirubicin, cisplatin, and fluorouracil as systemic treatment for advanced gastric carcinoma: a randomized phase II trial of the Swiss Group for Clinical Cancer Research. In J Clin Oncol. 2007;25(United States):3217-23.

6. Metzger R, Bollschweiler E, Holscher AH, Warnecke-Eberz U. ERCC1: impact in multimodality treatment of upper gastrointestinal cancer. Future Oncol. 2010;6:1735-49.

7. Wang D, Lippard SJ. Cellular processing of platinum anticancer drugs. Nat Rev Drug Discov. 2005;4:307-20.

8. Xu J, Yue CF, Zhou WH, Qian YM, Zhang Y, Wang SW, et al. Aurora-A contributes to cisplatin resistance and lymphatic metastasis in non-small cell lung cancer and predicts poor prognosis. J Transl Med. 2014;12:200.

9. Holohan C, Van Schaeybroeck S, Longley DB, Johnston PG. Cancer drug resistance: an evolving paradigm. Nat Rev Cancer. 2013;13:714-26.

10. Junttila MR, de Sauvage FJ. Influence of tumour micro-environment heterogeneity on therapeutic response. Nature. 2013;501:346-54.

11. Monte M, Benetti R, Buscemi G, Sandy P, Del Sal G, Schneider C. The cell cycle-regulated protein human GTSE-1 controls DNA damage-induced apoptosis by affecting p53 function. J Biol Chem. 2003;278:30356-64.

12. Collavin L, Monte M, Verardo R, Pfleger C, Schneider C. Cell-cycle regulation of the p53-inducible gene B99. FEBS Lett. 2000;481:57-62.

13. Utrera R, Collavin L, Lazarevic D, Delia D, Schneider C. A novel p53-inducible gene coding for a microtubule-localized protein with G2-phase-specific expression. Embo j. 1998;17:5015-25.

14. Monte M, Benetti R, Collavin L, Marchionni L, Del Sal G, Schneider C. hGTSE-1 expression stimulates cytoplasmic localization of p53. J Biol Chem. 2004;279:11744-52.

15. Scolz M, Widlund PO, Piazza S, Bublik DR, Reber S, Peche LY, et al. GTSE1 is a microtubule plus-end tracking protein that regulates EB1-dependent cell migration. PLOS ONE. 2012;7, e51259.

16. Hubner NC, Bird AW, Cox J, Splettstoesser B, Bandilla P, Poser I, et al. Quantitative proteomics combined with BAC TransgeneOmics reveals in vivo protein interactions. J Cell Biol. 2010;189:739-54.

17. Borner GH, Antrobus R, Hirst J, Bhumbra GS, Kozik P, Jackson LP, et al. Multivariate proteomic profiling identifies novel accessory proteins of coated vesicles. J Cell Biol. 2012;197:141-60.

18. Spanswick VJ, Lowe HL, Newton C, Bingham JP, Bagnobianchi A, Kiakos K, et al. Evidence for different mechanisms of 'unhooking' for melphalan and cisplatin-induced DNA interstrand cross-links in vitro and in clinical acquired resistant tumour samples. BMC Cancer. 2012;12:436.

19. Masica DL, Karchin R. Collections of simultaneously altered genes as biomarkers of cancer cell drug response. Cancer Res. 2013;73:1699-708.

20. Kim HK, Choi IJ, Kim CG, Kim HS, Oshima A, Michalowski A, et al. A gene expression signature of acquired chemoresistance to cisplatin and fluorouracil combination chemotherapy in gastric cancer patients. PLoS ONE. 2011;6, e16694.

21. Maeda O, Ando T, Ohmiya N, Ishiguro K, Watanabe O, Miyahara R, et al. Alteration of gene expression and DNA methylation in drug-resistant gastric cancer. Oncol Rep. 2014;31:1883-90.

22. Zeller C, Dai W, Steele NL, Siddiq A, Walley AJ, Wilhelm-Benartzi CS, et al. Candidate DNA methylation drivers of acquired cisplatin resistance in ovarian cancer identified by methylome and expression profiling. Oncogene. 2012;31:4567-76.

23. Tian T, Zhang E, Fei F, Li X, Guo X, Liu B, et al. Up-regulation of GTSE1 lacks a relationship with clinical data in lung cancer. Asian Pac J Cancer Prev. 2011;12:2039-43.

24. Cui J, Chen Y, Chou WC, Sun L, Chen L, Suo J, et al. An integrated transcriptomic and computational analysis for biomarker identification in gastric cancer. Nucleic Acids Res. 2011;39:1197-207.

25. D'Errico M, de Rinaldis E, Blasi MF, Viti V, Falchetti M, Calcagnile A, et al. Genome-wide expression profile of sporadic gastric cancers with microsatellite instability. Eur J Cancer. 2009;45:461-9.

26. Shen DW, Pouliot LM, Hall MD, Gottesman MM. Cisplatin resistance: a cellular self-defense mechanism resulting from multiple epigenetic and genetic changes. Pharmacol Rev. 2012;64:706-21.

27. Li M, Balch C, Montgomery JS, Jeong M, Chung JH, Yan P, et al. Integrated analysis of DNA methylation and gene expression reveals specific signaling pathways associated with platinum resistance in ovarian cancer. BMC Med Genomics. 2009;2:34. 
28. van Eijk KR, de Jong S, Boks MP, Langeveld T, Colas F, Veldink JH, et al. Genetic analysis of DNA methylation and gene expression levels in whole blood of healthy human subjects. BMC Genomics. 2012;13:636.

29. Mason EF, Rathmell JC. Cell metabolism: an essential link between cell growth and apoptosis. Biochim Biophys Acta. 2011;1813:645-54.

30. Hoefer J, Schafer G, Klocker H, Erb HH, Mills IG, Hengst L, et al. PIAS1 is increased in human prostate cancer and enhances proliferation through inhibition of p21. Am J Pathol. 2012;180:2097-107.

31. Ryan KM, Phillips AC, Vousden KH. Regulation and function of the p53 tumor suppressor protein. Curr Opin Cell Biol. 2001;13:332-7.

32. Norbury CJ, Zhivotovsky B. DNA damage-induced apoptosis. Oncogene. 2004;23:2797-808.

33. Wang L, Gao C, Yao S, Xie B. Blocking autophagic flux enhances matrineinduced apoptosis in human hepatoma cells. Int J Mol Sci. 2013;14:23212-30

34. Cui Q, Yu JH, Wu JN, Tashiro S, Onodera S, Minami M, et al. P53-mediated cell cycle arrest and apoptosis through a caspase-3- independent, but caspase-9-dependent pathway in oridonin-treated MCF-7 human breast cancer cells. Acta Pharmacol Sin. 2007;28:1057-66.

35. Hu W, Ge Y, Ojcius DM, Sun D, Dong H, Yang XF, et al. p53 signalling controls cell cycle arrest and caspase-independent apoptosis in macrophages infected with pathogenic Leptospira species. Cell Microbiol. 2013;15:1642-59.

36. Bublik DR, Scolz M, Triolo G, Monte M, Schneider C. Human GTSE-1 regulates p21(CIP1/NAF1) stability conferring resistance to paclitaxel treatment. J Biol Chem. 2010;285:5274-81.

37. Abbas T, Dutta A. p21 in cancer: intricate networks and multiple activities. Nat Rev Cancer. 2009;9:400-14.

\section{Submit your next manuscript to BioMed Central and take full advantage of:}

- Convenient online submission

- Thorough peer review

- No space constraints or color figure charges

- Immediate publication on acceptance

- Inclusion in PubMed, CAS, Scopus and Google Scholar

- Research which is freely available for redistribution 